\title{
Polyunsaturated fatty acids in marine bacteria and strategies to enhance their production
}

\begin{abstract}
Polyunsaturated fatty acids (PUFAs) play an important role in human diet. Despite the wideranging importance and benefits from heart health to brain functions, humans and mammals cannot synthesize PUFAs de novo. The primary sources of PUFA are fish and plants. Due to the increasing concerns associated with food security as well as issues of environmental contaminants in fish oil, there has been considerable interest in the production of polyunsaturated fatty acids from alternative resources which are more sustainable, safer, and economical. For instance, marine bacteria, particularly the genus of Shewanella, Photobacterium, Colwellia, Moritella, Psychromonas, Vibrio, and Alteromonas, are found to be one among the major microbial producers of polyunsaturated fatty acids. Recent developments in the area with a focus on the production of polyunsaturated fatty acids from marine bacteria as well as the metabolic engineering strategies for the improvement of PUFA production are discussed.
\end{abstract}

Keyword: Docosahexaenoic acid (DHA); Eicosapentaenoic acid (EPA); Marine bacteria; Metabolic engineering; Polyunsaturated fatty acids 